\title{
Letter
}

\section{Prototype Portable Full-color LED Illumination Panel and Palm-sized Controller}

\author{
Shigeharu TAMURA*, Takashi HIRAGA** and Hideo SUGIMOTO*** \\ *National Institute of Advanced Science and Technology (AIST), Health Research Institute \\ **National Institute of Advanced Science and Technology (AIST), Electronics and Photonics Research Institute \\ ***DAINICHI DENSHI CO., LTD
}

Received July 31, 2012, Accepted October 2, 2012

Paper originally published in Japanese in J. Color Science Association of Japan, Vol.35, supplement, pp.40-41 (2011).

\begin{abstract}
A portable-sized full-color LED illumination panel and a Palm-sized Controller were developed. In addition to 3 primary color (red, green, and blue) LED, white LED was used in order to achieve high illuminance in the white illumination region and to facilitate realization of pastel colors. A mobile computer was used as the controller. Adjustment of illuminance is performed through the duty ratio control device connected to the computer. By using this controller system, 4 types of LED could be controlled according the input 3 information (Chromaticity Coordinates and light intensity) by a real-time calculation. Up to 255 illumination panels can be connected. This system allows individual control of a maximum of 255 illumination panels, or control of all illumination panels using the same illumination conditions. By using this system, 9 types of illumination colors were selected and some characteristics were measured. For every illumination, the chromaticity coordinates hardly changed above the duty ratio above $30 \%$.
\end{abstract}

KEYWORDS: LED, color LED, full-color, portable light, palm-sized controller

In recent years, many kinds of color LED light products have been sold for the various purposes. These products, however, have some points which should be improved: for example, the choice of a color or brightness and the convenience of a controller are not necessarily enough. The authors, therefore, developed a new full-color LED illumination panel.

The size of the LED illumination panel is $30 \mathrm{~cm} \times 30$ $\mathrm{cm} \times 5 \mathrm{~cm}$ (portable size). In order to achieve high illuminance in the white illumination region that is used in daily life and to facilitate realization of pastel colors, white LED was also used in addition to 3 primary colors of light (red, green, and blue LED). A total of 900 LED (circular type) was used: 400 red LED with the peak wavelength of $630 \mathrm{~nm}$ (SLI580UT3F, ROHM Co., Ltd.), 200 green LED with that of $518 \mathrm{~nm}$ (SLA580ECT3F, ROHM Co. Ltd.) 200 blue LED with that of $468 \mathrm{~nm}$ (SLA580BCT3F, ROHM Co., Ltd.) and 100 white LED with chromaticity coordinate of $x=0.30, y=0.32$ (Nichia CORPORATION). A sheet of diffuser with the thickness of $0.3 \mathrm{~mm}$ was set at the front. Before developing the LED light source, the measured peak wavelengths of each LED were measured: $635 \mathrm{~nm}$ for red, $523 \mathrm{~nm}$ for green, $465 \mathrm{~nm}$ for blue and both $470 \mathrm{~nm}$ and $550 \mathrm{~nm}$ for white.

Color and brightness, which a user wishes, can be controlled by touching a point on the chromaticity dia-

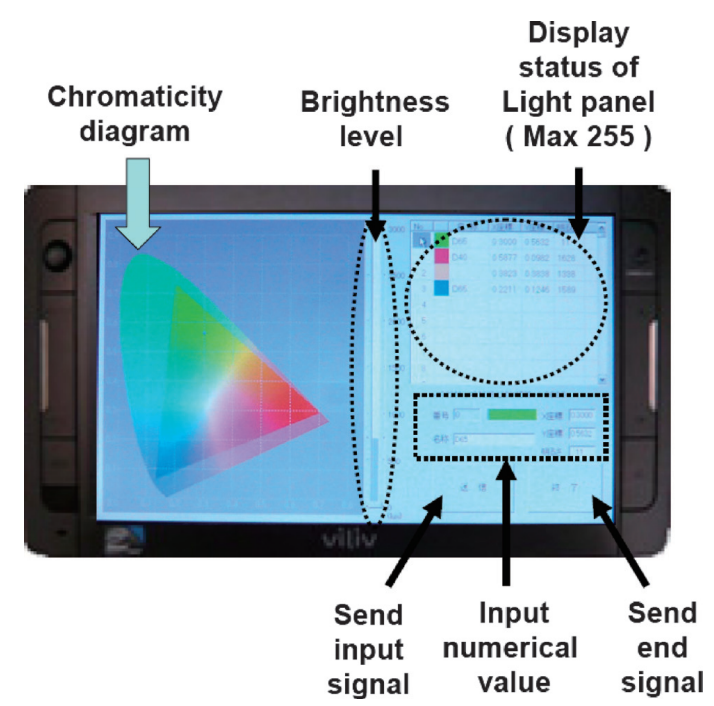

Figure 1 Palm-sized mobile computer for color light

gram displayed on a palm-sized mobile computer screen as well as brightness level bar, or three values can be entered in the table displayed on the screen and touching the "send" button (Figure 1). That is, three types of input from the display will allow real-time calculation of brightness of 4 types of LED, and control the colors. Adjustment of illuminance is performed through the duty ratio control device connected to the computer 


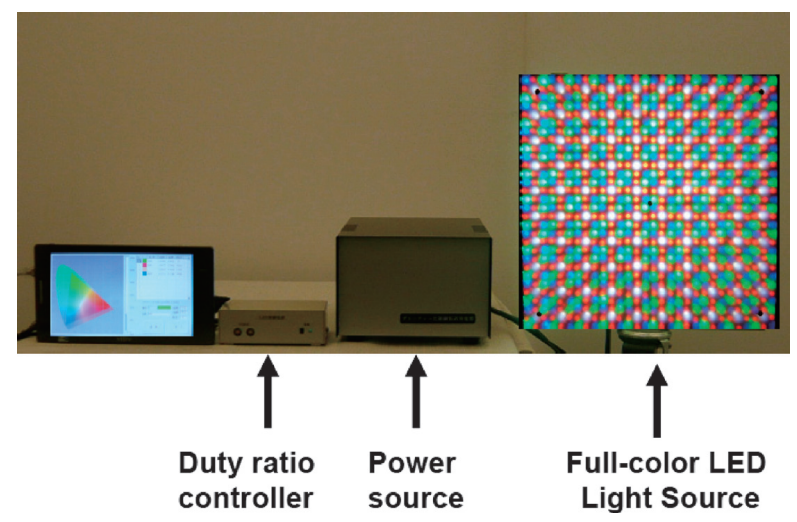

Figure 2 Portable full-color LED illumination panels and palmsized controller

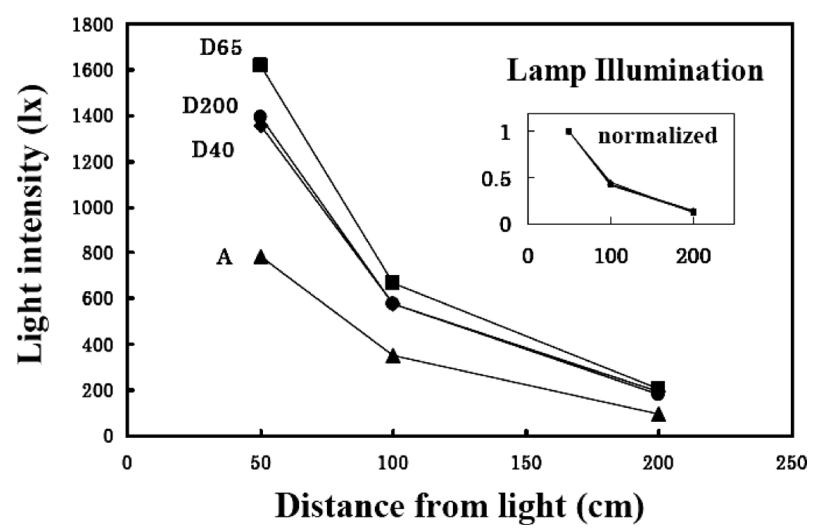

Figure 3 Relation of illuminance and distance from light source (4 light illuminations)

through a USB-RS485 conversion cable. The program which calculates this nonlinear equation is described in reference (1). This computer system allows individual control of a maximum of 255 illumination panels, and control of all illumination panels using the same illumination conditions. Usage of illumination requires calibration suitable for the environment (specifically based on distance from the light source), and another control screen specifically for this purpose is provided on the computer. Light each LED separately at maximum illuminance, measure its chromaticity and illuminance, and enter this data on the display. This operation is also effective for calibration when the characteristics of the LED have changed over time. The photograph of the color LED lighting system is shown in Figure 2.

Some optical properties of this color LED light source were examined. Nine types of illumination patterns were selected: 5 colored illuminations (red, green, blue, yellow and purple) and 4 lamp illuminations along the blackbody locus (A, D40, D65 and D200). The chromaticity coordinates of colored illuminations are $x=0.55$, $y=0.30$ for red, $x=0.25, y=0.60$ for green, $x=0.20, y=0.20$

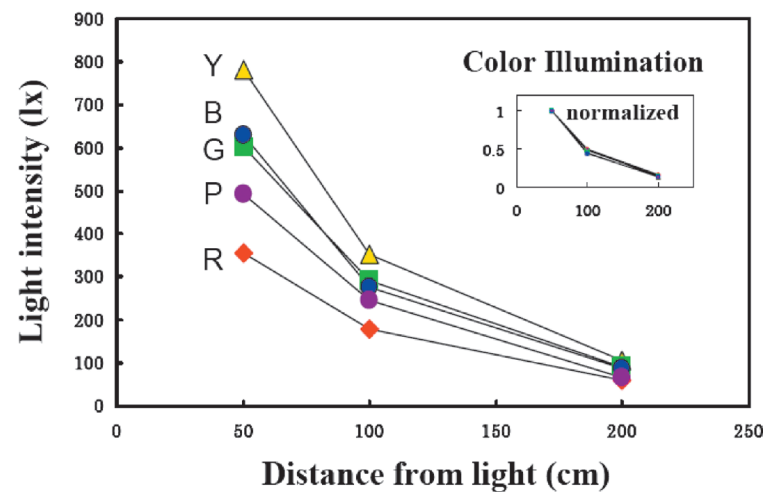

Figure 4 Relation of illuminance and distance from light source (5 colored illuminations)

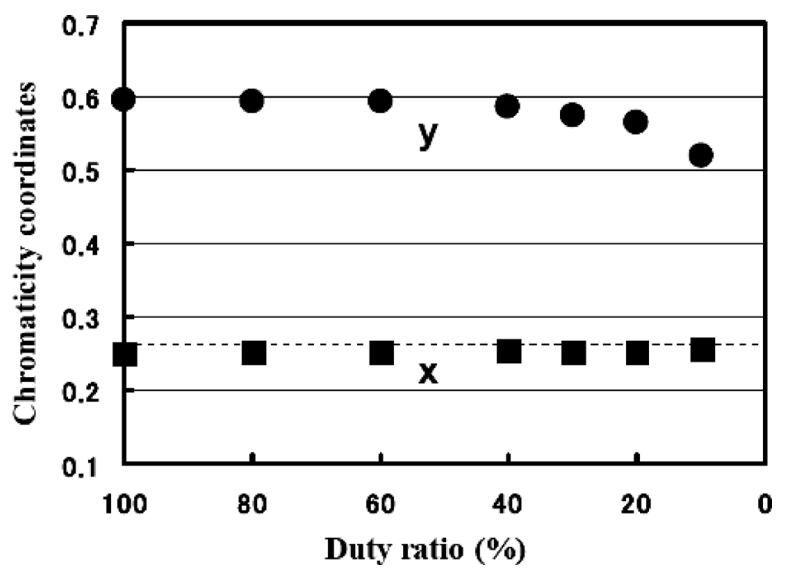

Figure 5 Relation between chromaticity coordinates and illuminance (duty ratio) of green LED

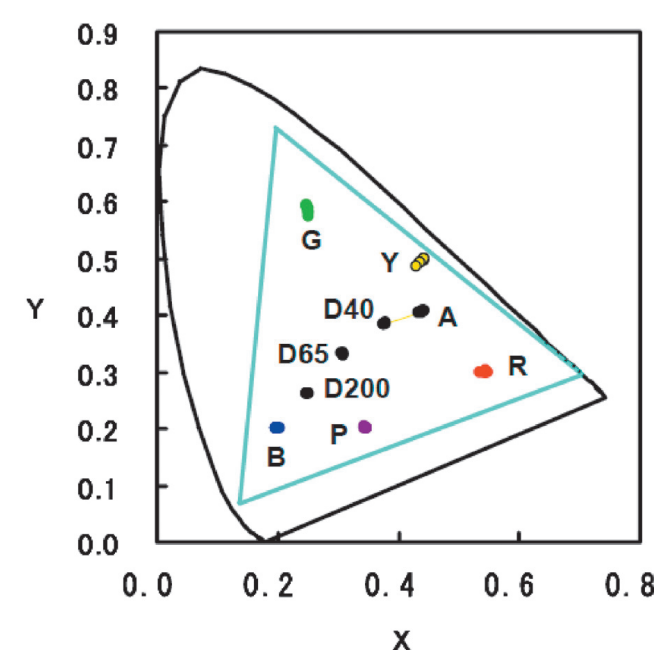

Figure 6 Relation between chromaticity coordinates and illuminance at chromaticity diagram with duty ratio above $30 \%$ 
for blue, $x=0.45, y=0.50$ for yellow and $x=0.35, y=0.20$ for purple.

The relation of the illuminance and the distance from the illumination panel is shown in Figure 3 and Figure 4, respectively. Each normalized graph is also shown. For all types of illumination patterns, similar normalized patterns were obtained.

The relation between the chromaticity coordinates and the illuminance were measured. For all types of illumination patterns, the coordinates hardly changed above the duty ratio $30 \%$. Below $20 \%$, significant differences are observed like Figure 5. The reason will be as followings. The chromaticity coordinates for all illumination patterns were also with the duty ratio above $30 \%$ shown in Figure 6. Duty control was performed by 256 step values at this system. The values for each kind of LED (red, green, blue and white) are determined by the real-time calculation so that the characteristic of the user's input data (chromaticity coordinates and brightness) is satisfied. These step values can be seen on the display. The duty ratio below $20 \%$, it becomes marked to determine the optimum step values. As the results, it is difficult to realize optimum characteristic of the light. In order to avoid such a phenomenon, a high-performance element (such as a 16 bit processor) should be used.

In conclusion, a portable full-color LED illumination panel and a palm-sized mobile a controller were developed. Color and brightness, which a user wishes, can be controlled very easily and quickly. This LED light system can be used as the stage illumination. Also this controller (software) will be useful in the field of the plant factory, the medical service such as the detection of the involved skin.

\section{Referfences}

(1) Takahama, K., Hiraga, T. and Tamura, S.: Japan patent 4565099 (13 August 2010). 